\title{
Searching for civilization
}

Over the past half century, efforts to find evidence of life elsewhere in the Universe have centred on the search for so-called technosignatures - signs of technology being employed by a civilization that 'modifies its environment in ways that are detectable.' For example, our current knowledge of physics suggests there are strong limits to the frequency spread of narrow-band radio-frequency emissions. The observation of a signal violating such limits would present evidence of some intelligence that created it. A few potential signals of this kind have been seen - notably, a 72-second signal detected in 1977 coming from the Sagittarius constellation, known since then as the 'WOW!' signal, after the exclamation written onto a print out of the recording by astronomer Jerry Ehman. Its interpretation remains uncertain, as subsequent searches have failed to record it again.

Apart from narrow-band signals, researchers have also made systematic searches for other technosignatures such as pulsed radio waves and laser emissions. An intelligent civilization may also build structures to harvest the energy of a star, and, if operating on a sufficient scale, could detectably alter the light coming from it. Energy use on a large scale may also leave signs of waste heat. So far, however, searches for all such signatures have come up empty. We might really be alone.

Even so, our basic understanding even of electricity and magnetism is still incomplete, and our knowledge of the range of potential future technologies little more than speculation. The scope of our observations to date is also extremely limited, even though the sensitivity of telescopes has improved by some 14 orders of magnitude over the past 50 years. In the case of continuous radiofrequency signals, for example, researchers have made estimates of the fraction of the spectrum and possible space directions we've explored within the relatively quiet 1 to $10 \mathrm{GHz}$ range - the so-called water hole - considered to be a spectral region that is likely to be preferred by any intelligence trying to make contact with us. The resulting fraction is around $10^{-22}$. We have so far looked at almost nothing.

The current status of the search for technosignatures was the focus of a NASA meeting in September 2018, the main points reviewed in a recent paper (NASA Technosignatures Workshop Participants, preprint at https://arxiv.org/abs/1812.08681; 2018). As the report makes clear, the search rests heavily on imagination to conceive of the kinds of possible technologies a civilization far more advanced than our own may develop. They could well be mostly invisible to us, operating in a regime of physics we as yet know nothing about. And our search so far based even on known physics is extremely limited.

As the authors note, until we actually detect a genuine technosignature, research progress will consist of finding improved and more sensitive ways to search for technosignatures, and the progressive establishment of ever stronger upper limits on specific signatures. Astronomers have established weak upper limits not only on continuous radio-frequency radiation, but also on the detection of waste heat, possible stellar obstruction by large engineered structures, and for the influence of so-called Dyson Spheres - hypothetical structures built to enclose a star and potentially capture a significant portion of its radiated energy. These limits will be made stronger in coming years.

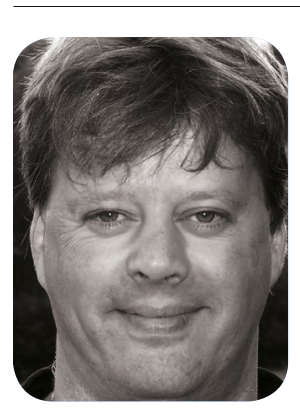

Over the past half century, efforts to find evidence of life elsewhere in the Universe have centred on the search for so-called technosignatures.

Another important avenue involves the search within our own Solar System for objects such as interstellar probes that might have been sent here from other civilizations. In 2017, the elongated asteroid Oumuamua roughly 1,000 metres in length - was the first macroscopic body of interstellar origin ever found within the Solar System, moving so rapidly relative to the Sun that it could not have originated within the Solar System. We have little information on its nature, although it is metal-rich and of relatively high density, consistent with a possible technological origin. Astronomers expect there should be several such objects of natural origin passing within Earth's orbit each year. Improved techniques to search for such 'interlopers' will, if nothing else, lead to better understanding of such natural objects and their origins.

Indeed, as the authors of the NASA review point out, this is true of the search for all types of technosignatures, illustrating a research philosophy articulated many years ago by Freeman Dyson: "Every search for alien civilizations should be planned to give interesting results even when no aliens are discovered."

Even if many other intelligent civilizations do exist, we should probably expect a long time to pass before we discover any sign of their technological use. We are a very young civilization ourselves. A notable point made by the NASA report is that our greatest limitation may come in trying to predict the likely paths of biological, cultural and technical evolution of civilizations as they grow far beyond our current stage of development. A civilization advanced far beyond us in technology may find it especially easy to fool us by disguising its technosignatures to look as if they have natural origins.

But we may find that some features of civilization and physics will inevitably lead to prominent technosignatures. For example, if social development for all civilizations runs along similar lines, we may expect any civilization to possess centres of social and technological concentration - cities - into which waste heat will be strongly focused, raising local temperatures. Infrared imaging from space can readily detect such 'heat islands' on Earth already. All civilizations will also likely rely on communications, which will have some leakage into space. The proposed Square Kilometer Array radio telescope project will in coming years be sensitive enough to detect Earth 2020 levels of radio 'leakage' at interstellar distances.

Another signature to search for is the artificial alteration of planetary characteristics, in analogy to the way humanity has altered the Earth's surface, atmospheric and biological character. If many civilizations face a stage of possible technological collapse - as we may be facing soon on Earth - there may be atmospheric technosignatures that would be detectable in the final stages. For example, planetary warming is likely a challenge faced by any civilization harnessing everincreasing amounts of energy. Sensitive enough instruments may ultimately show that other cultures have faced this particular problem long before us.

\section{Mark Buchanan}

Published online: 1 February 2019 https://doi.org/10.1038/s41567-019-0423-X 